\title{
Dialogue Concerning Two Views on Quantum Coherence: Factist and Fictionist
}

\author{
Stephen D. Bartlett, ${ }^{1}$ Terry Rudolph, ${ }^{2,3}$ and Robert W. Spekkens ${ }^{4}$ \\ ${ }^{1}$ School of Physics, The University of Sydney, New South Wales 2006, Australia \\ ${ }^{2}$ Optics Section, Blackett Laboratory, Imperial College London, London SW7 2BW, United Kingdom \\ ${ }^{3}$ Institute for Mathematical Sciences, Imperial College London, London SW7 2BW, United Kingdom \\ ${ }^{4}$ Perimeter Institute for Theoretical Physics, 31 Caroline St. N, Waterloo, Ontario N2L 2Y5, Canada
}

(Dated: 31 October 2005)

\begin{abstract}
A controversy that has arisen many times over in disparate contexts is whether quantum coherences between eigenstates of certain quantities are fact or fiction. We present a pedagogical introduction to the debate in the form of a hypothetical dialogue between proponents from each of the two camps: a factist and a fictionist. A resolution of the debate can be achieved, we argue, by recognizing that quantum states do not only contain information about the intrinsic properties of a system but about its extrinsic properties as well, that is, about its relation to other systems external to it. Specifically, the coherent quantum state of the factist is the appropriate description of the relation of the system to one reference frame, while the incoherent quantum state of the fictionist is the appropriate description of the relation of the system to another, uncorrelated, reference frame. The two views, we conclude, are alternative but equally valid paradigms of description.
\end{abstract}

This paper is dedicated to the memory of Asher Peres. Asher had thought about and discussed with one of us many of the issues we address here and had planned a paper of his own on the subject. We will miss greatly the insight, clarity and intellectual honesty he could bring to bear on the deepest conceptual problems in physics.

\section{INTRODUCTION}

We shall be considering a debate in quantum theory that has arisen many times in many different contexts. It is the debate over whether it is possible to prepare states that are coherent superpositions of eigenstates of certain observables. One can characterize the two camps by opposing slogans: "coherence as fact" versus "coherence as fiction". Implicit in much of the discussion is the assumption that quantum states directly describe the intrinsic properties of a system and consequently that there is a matter of fact about whether or not such coherences exist.

We shall suggest the following resolution to this debate: whether or not it is appropriate to assume quantum coherences in the state assignment for some system depends on the external reference frame with respect to which that system is being described. Specifically, it depends on whether this reference frame is correlated with the system or not. We shall argue that the two sorts of descriptions are both valid and consequently that the presence or absence of coherences between eigenstates of certain observables is not a matter of fact, but rather depends on one's conventional choice of reference frame. Central to our argument is establishing the consistency of two descriptions: one where the reference frame is treated internally, in the sense of receiving representation within the Hilbert space formalism, and another where it is treated externally, as a classical system. Our position is by no means a new one; it has many precursors in the literature, in particular in the work of Aharonov and
Susskind [1]. We hope, however, that the analysis presented herein will illuminate and add to what has come before.

\section{THE DEBATE}

One context in which the debate over quantum coherence arises is superconductivity, where there has been disagreement about whether the Bardeen-Cooper-Schrieffer ground state, which involves a coherent superposition of different charge eigenstates, is the actual state of a superconductor or whether the coherence is merely a mathematical convenience 2, 3, 4]. The same argument arises in the context of Bose-Einstein condensation regarding coherent superpositions of different atom number eigenstates $5,6,7,6$. In both cases, standard practice in the condensed matter community is to assign an order parameter to the condensate, which is typically defined as the expectation of the quantum field operator. Thus, if the quantum state has no coherence between different eigenstates of the number of Cooper pairs or atoms, the order parameter is zero. However, the usefulness of the concept of a non-zero order parameter suggests that there is something wrong with this approach. The prediction [5] and subsequent observation [9] of the interference of independent atomic condensates has also fueled the debate on phase coherence in these systems.

The debate has also arisen in the context of quantum optics, where the issue is the existence of coherence between eigenstates of different photon number. This is the forum in which the debate has seen the most recent activity (although an early version of it can be found in Refs. 10, 11, 12]). It is also perhaps the context in which the debate has been the most sophisticated, due to the advanced techniques for describing and implementing generalized measurements and state preparations now commonly employed by the quantum optics community. Also, in as much as the physical descriptions in quantum 
optics can be directly derived from a fundamental theory (Quantum Electrodynamics) without recourse to effective theories, as in the Bose-Einstein condensation and superconductivity examples, one might have expected that such controversies can be rigorously settled one way or the other. We will focus on the optical context here for concreteness.

Recent interest in the optical version of the debate begins with the 1997 paper by Klaus Mølmer entitled "Optical coherence: a convenient fiction" 13]. The standard assumption in the quantum optics community is that a laser operating above threshold emits an electromagnetic (EM) field for which the quantum state is

$$
|\alpha\rangle=\sum_{n=0}^{\infty} \frac{e^{-|\alpha|^{2} / 2} \alpha^{n}}{\sqrt{n !}}|n\rangle
$$

where $\alpha$ is complex. This is known as a Glauber state or coherent state. It is a coherent superposition of photon number eigenstates with a phase that varies linearly with number, and number-state populations that obey a Poissonian distribution. In other words, if $\alpha=\sqrt{n} e^{i \phi}$, then the relative phase between $|n+k\rangle$ and $|n\rangle$ is $e^{i k \phi}$, and the probability of $n$ photons is

$$
p_{n}=\frac{e^{-\bar{n}} \bar{n}^{n}}{n !} .
$$

In his paper, Mølmer tries to cast doubt on this assumption about the state of a laser by considering how a laser field is produced.

His argument relies on the following assumptions: (i) the atoms of the gain medium are treated quantum mechanically, (ii) these atoms are initially described by an incoherent mixture of energy eigenstates, and (iii) energy is conserved in the interaction between the atoms and the optical field. With these assumptions, the interaction between an atom in the gain medium and the electromagnetic field is such that, if the atom is excited and the field is initially described by an $n$ photon eigenstate, then the atom+field evolves over a time $t$ to a coherent superposition of what one started with and a state wherein the atom has de-excited and the field has acquired an additional photon,

$$
|e\rangle|n\rangle \rightarrow a(t)|e\rangle|n\rangle+b(t)|g\rangle|n+1\rangle,
$$

where $a(t), b(t)$ are complex amplitudes. Note that this state is pure and entangled. If one is interested only in the reduced density operator of the field, obtained by taking the trace over the atom, one finds that the state of the field is an incoherent mixture of $n$ and $n+1$ photons,

$$
\rho=|a(t)|^{2}|n\rangle\left\langle\left. n|+| b(t)\right|^{2} \mid n+1\right\rangle\langle n+1| .
$$

The gain medium of the laser as a whole is simply an incoherent sum of different numbers of excitations, each term of which evolves to an entangled state between the gain medium and the field. A careful analysis [13] shows that the reduced density operator of the field is found to be of the form:

$$
\rho=\sum_{n=0}^{\infty} p_{n}|n\rangle\langle n|,
$$

with $p_{n}$ the Poissonian distribution of Eq. (2). Thus, although the populations of the number states are what we expected (the same as for the coherent state), there are no coherences, and thus no phase relations, between these. Thus, surprisingly, Mølmer's account of the inner workings of the laser seems to imply that the field emitted by a laser operating above threshold is not the coherent state of Eq. (1) as is usually assumed, but rather the incoherent state of Eq. (5).

Mølmer concludes that the coherence was just a fiction. Maybe it is convenient to assume, maybe one doesn't make mistakes by assuming it, but it isn't really there.

\section{A POSSIBLE DIALOGUE}

Subsequent to this, there was a flurry of activity on the subject [14, 15, 16, 17, 18, 19, 20, 21, 22, 23, 24, 25, 26]. In addition to what has appeared in the literature, there have been a great number of debates on this issue at various conferences and among workers in the field, which supplement the arguments found in the literature. We have ourselves benefitted a great deal from early discussions with John Sipe, and ongoing discussions with Barry Sanders and Howard Wiseman on this subject. There is much to learn from the details of these debates. Note that we do not attempt to provide a historically accurate account of the relevant literature or of who believed what at various stages of the debate. Rather, we shall try to simply give a flavour of the argument and the central issues. We therefore present the debate in the form of a hypothetical dialogue between purists from the two camps. This dialogue is representative of many of the arguments and counterarguments that have been provided. ${ }^{1}$

We shall call the proponent of the idea that coherence is fact "the factist" and the proponent of the idea that coherence is fiction "the fictionist". We join the story at the stage where the fictionist is just finishing the argument we described above.

Fictionist: [...] And so you see, if you do a proper quantum analysis of the manner in which laser light is produced, you find that the reduced density operator of the field is an incoherent sum of number states, not a coherent superposition. Optical coherence is a fiction!

Factist: It's a cute argument, and I admit that I had to give it some thought before I saw what was wrong with

\footnotetext{
1 The arguments for both sides of the debate are sufficiently compelling that it is easy to find oneself (and indeed we have found ourselves) defending different positions at different times.
} 
it, but I've figured it out. The key is that the reduced density operator can be written as an incoherent sum of number states, but it can also be written as an incoherent sum of coherent states. That is, expressing $\alpha=|\alpha| e^{i \phi}$ in polar coordinates, with $\phi$ the phase of the coherent state $|\alpha\rangle=\| \alpha\left|e^{i \phi}\right\rangle$, then we can rewrite Eq. (5) as

$$
\rho=\sum_{n=0}^{\infty} p_{n}|n\rangle\left\langle n\left|=\int_{0}^{2 \pi} \frac{d \phi}{2 \pi}\right||\alpha| e^{i \phi}\right\rangle\left\langle|\alpha| e^{i \phi}\right| .
$$

As a result, we can interpret the situation as follows: the field is actually in some particular coherent state $|\alpha\rangle$, we just don't know which, because we don't know a priori what is the phase of the laser. As a result of this ignorance, we have to represent our knowledge by an incoherent sum of coherent states equally weighted over all phases. So the old way of looking at this was right all along. There really is coherence there.

Fictionist: Look, I know all about this multiplicity of convex decompositions of a mixed state, sometimes called the "ambiguity of mixtures" [27, 28], but you're wrong to assign special significance to one such decomposition because one cannot adopt an ignorance interpretation of an improper mixed state. ${ }^{2}$ Here's the problem. If you tell me that really the quantum state of the field is $|\alpha\rangle$ for some $\alpha$, then you're telling me that really the reduced density operator for the field is $|\alpha\rangle\langle\alpha|$ and the only quantum state of the atoms +field that has this as a reduced density operator is a product state of the form $|\chi\rangle|\alpha\rangle$ for some atomic state $|\chi\rangle$. But then you are saying that really the atoms+field system is in a product state, and this contradicts the assumption we started with, that the atoms+field system is in an entangled state.

Factist: I suppose I hadn't thought that through carefully enough. But now that I have, I realize what the correct response is. One of your assumptions was that the gain medium of the laser was in an incoherent mixture of energy eigenstates, but you're wrong. It's actually in a coherent superposition of energy eigenstates. Roughly speaking, the lasing phase transition occurs because the atoms start oscillating in phase with each other due to a symmetry breaking which occurs when stimulated emission (which preserves phase) dominates spontaneous emission - and we must therefore describe the atomic state $|\phi\rangle$ as one depending on the common phase $\phi$ of their oscillation. Simple mean field theory descriptions of the symmetry breaking accompanying this transition [30, 31] show that the state of the atoms is one which involves a non-zero expectation value of the atomic dipole moment operator, which in turn implies that their state involves a coherent superposition of energy eigenstates. The standard atom-field interaction serves to transfer

\footnotetext{
2 An improper mixture is one that arises as the reduced density operator of a pure entangled state, while a proper mixture is one that arises as an incoherent sum of pure states [29].
}

this coherence to the emitted field - that is why there is a nonvanishing expectation of the annihilation operator for the field.

Fictionist: Even if the gain medium had a welldefined phase, you don't know what it is, so you have to describe it by the state that is a mixture over all phases,

$$
\begin{aligned}
\rho_{\text {atoms }} & =\int_{0}^{2 \pi} \frac{d \phi}{2 \pi}|\phi\rangle\langle\phi| \\
& =\sum_{n} w_{n}\left|E_{n}\right\rangle\left\langle E_{n}\right|,
\end{aligned}
$$

where $\left|E_{n}\right\rangle$ are the energy eigenstates of the gain medium and $w_{n}$ is some probability distribution. It is $\rho_{\text {atoms }}$ that you should use in the calculation, and because this state has a vanishing dipole moment, you can't develop any coherence in the field, as I showed before.

Factist: The density operator $\rho_{\text {atoms }}$ might be fine for calculations, but what's actually going on is that there is some pure state $|\phi\rangle$ that describes the atoms.

Fictionist: [groan] Haven't we been over this before? For you to interpret $\rho_{\text {atoms }}$ as a mixture of the states $|\phi\rangle$ rather than a mixture of the states $\left|E_{n}\right\rangle$ is to favor one convex decomposition over another, which is a fallacy!

Factist: Not this time! It's only a fallacy if the system in question is really entangled with something else, which is not the case for the atoms of the gain medium.

Fictionist: Well, really, the gain medium is prepared by some pumping mechanism, and we need to treat the electrons of this mechanism quantum mechanically. Because they start out in a proper mixture of energy eigenstates with no dipole moment and because the interaction with the atoms of the gain medium is energy-conserving to good approximation, we find that in the end the electrons and the atoms are in a proper mixture of entangled states. Each such entangled state is a coherent superposition of different ways of distributing the energy between the pumping mechanism and the gain medium and the reduced density operator on the atoms for each is an improper mixture. So to claim that one convex decomposition of $\rho_{\text {atoms }}$ is preferred is indeed a fallacy.

Factist: I question your assumption about the initial state of the pumping mechanism, but I'm going to drop it because it seems to me that the question of whether or not there exists coherence should ultimately be settled by experiment, and unfortunately for you, the experiments show that the fields emitted by lasers are in coherent states.

The experiment I'm thinking of is a simple balanced homodyne detection [shown in Fig. 11. One mixes the signal (mode $a$ ) with a local oscillator (mode $b$ ) at a 50/50 beam splitter and detects the difference in the intensity at the two output ports (modes $c$ and $d$ ). The signal is given a variable phase shift of $\phi$ prior to the beam splitter, and the difference in intensity is measured as a function of this phase shift.

Suppose that the path lengths are arranged such that for $\phi=0$ one finds the mean intensity at the two output 


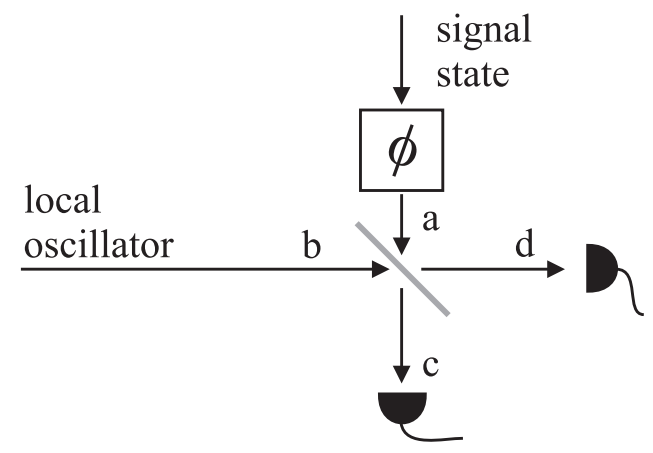

FIG. 1: Factist's schematic for homodyne detection of a signal state using a local oscillator, a beamsplitter, and photodetectors. A variable phase shift $\phi$ can be applied to the signal state.

ports to be equal. If the signal mode is initially described by the density operator $\rho$, then after a phase shift of $\phi$, it is described by $e^{i \phi N} \rho e^{-i \phi N}$ where $N$ is the number operator. Let $a$ denote the annihilation operator for the signal mode, let $\beta$ denote the classical electromagnetic field for the local oscillator, and let $c$ and $d$ denote the annihilation operations for the two output modes. By the action of the beam splitter,

$$
c=\frac{1}{\sqrt{2}}(a-\beta) \quad d=\frac{1}{\sqrt{2}}(a+\beta) .
$$

The Hermitian operator associated with the difference in intensity at the output ports is 32

$$
d^{\dagger} d-c^{\dagger} c=\left(\beta^{*} a+\beta a^{\dagger}\right)
$$

Thus, for a quantum state $\rho$ that suffers a phase shift of $\phi$ we expect an intensity difference of

$$
\begin{aligned}
I_{d}-I_{c} & =\operatorname{Tr}\left(e^{i \phi N} \rho e^{-i \phi N}\left(\beta^{*} a+\beta a^{\dagger}\right)\right) \\
& =\operatorname{Tr}\left(\rho\left(\beta^{*} e^{-i \phi} a+\beta e^{i \phi} a^{\dagger}\right)\right) .
\end{aligned}
$$

Interference, that is, variation of $I_{c}-I_{d}$ with $\phi$, can only arise if $\operatorname{Tr}(\rho a)$ is non-zero, that is, if $\rho$ has coherence between different number eigenstates.

Thus, seeing interference in a homodyne detection measurement demonstrates the presence of coherence. The experiment has been done for a coherent state signal, $\rho=|\alpha\rangle\langle\alpha|$, and the interference is observed. So experiment shows that coherences exist.

Fictionist: [Shaking her head] You're always forgetting to think a bit about where these systems (such as your "local oscillator") come from! The experiment actually looks like Fig. 2

The local oscillator and the signal are not independent - they come from a common source! So really what happens is that the source is beat against the vacuum at an unbalanced beam splitter with transmission probability $T$. If the source is a Fock state $|n\rangle$, then after the first of

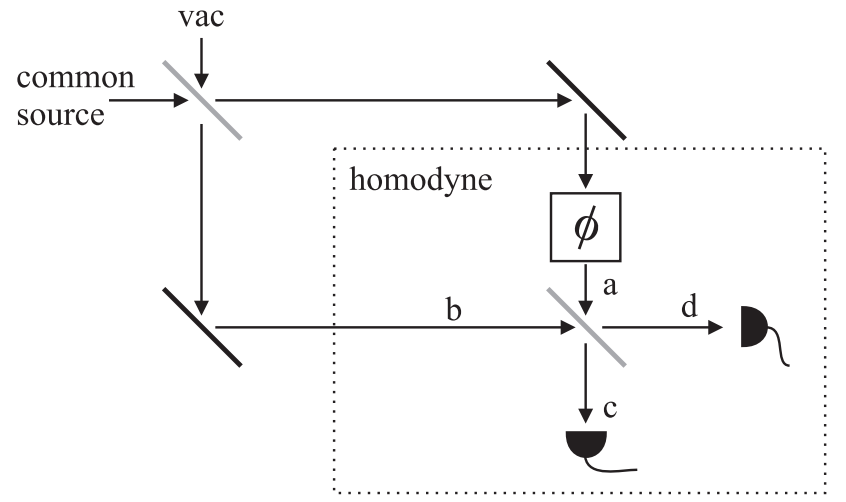

FIG. 2: Fictionist's schematic for homodyne detection wherein the local oscillator arises from the same common source as the signal.

the two beam splitters, the state of modes $a$ and $b$ is 33 .

$$
\left|\psi_{n}\right\rangle=\sum_{m} c_{m}^{(n)}|m\rangle|n-m\rangle
$$

where

$$
c_{m}^{(n)}=2^{-n / 2} \sqrt{\left(\begin{array}{c}
n \\
m
\end{array}\right)} T^{m / 2}(1-T)^{(n-m) / 2} .
$$

The phase shift by $\phi$ causes the quantum state to evolve to

$$
\left|\psi_{n, \phi}\right\rangle=\sum_{m} c_{m}^{(n)} e^{-i \phi m}|m\rangle|n-m\rangle,
$$

But note that the reduced density operator for the signal is still of the form

$$
\rho=\operatorname{Tr}_{b}\left|\psi_{n, \phi}\right\rangle\left\langle\left.\psi_{n, \phi}\left|=\sum_{m}\right| c_{m}^{(n)}\right|^{2} \mid m\right\rangle\langle m|
$$

which is devoid of coherence.

It is of course more realistic to assume that the source puts out a Poissonian mixture of Fock states, but then after the beam splitter and phase shifter, the state is

$$
\rho_{a b}=\sum_{n} p_{n}\left|\psi_{n, \phi}\right\rangle\left\langle\psi_{n, \phi}\right|
$$

The reduced density operator for the signal mode is

$$
\begin{aligned}
\rho=\operatorname{Tr}_{b}\left(\rho_{a b}\right) & =\sum_{m}\left(\sum_{n} p_{n}\left|c_{m}^{(n)}\right|^{2}\right)|m\rangle\langle m| \\
& =\sum_{m} p_{m}|m\rangle\langle m|
\end{aligned}
$$

where $p_{m}$ is a Poissonian distribution (the proof of this is straightforward using the techniques of Ref. 21] ). Thus, the state of the signal is incoherent. 
Despite this incoherence, one still predicts interference. Denoting the annihilation operator associated with the local oscillator by $b$, we have

$$
c=\frac{1}{\sqrt{2}}(a-b), \quad d=\frac{1}{\sqrt{2}}(a+b) .
$$

The Hermitian operator corresponding to the relative number of photons found at the two detectors is therefore

$$
d^{\dagger} d-c^{\dagger} c=a^{\dagger} b+b^{\dagger} a
$$

It is easy to verify that $\left\langle\psi_{n, \phi}\left|a^{\dagger} b+b^{\dagger} a\right| \psi_{n, \phi}\right\rangle \propto \sin \phi$. Thus, even the state $\rho_{a b}$ shows interference, because every term in the incoherent sum is proportional to $\sin \phi$.

So, the interference is explained by the fact that one has coherence between different ways of distributing $n$ photons between a pair of modes, not by the fact that one has coherence between different numbers of photons in a single mode.

Factist: Well, I admit I can't see any mistake in what you've done, but I'm still not convinced. Hasn't it been shown experimentally [34] that interference is obtained even between two independent lasers?

Fictionist: Yes, but the interference that is observed can still be explained without needing to invoke coherence. Let me convince you of this using the simplest example of a pair of Fock states. Suppose that initially the state is $|n\rangle|n\rangle$. The output port in which the first photon is detected is completely random. However, after this detection, the state must be updated to $(1 / \sqrt{2})(|n-1\rangle|n\rangle \pm|n\rangle|n-1\rangle)$ with the relative phase being fixed by the random outcome of the first detection. After many such detections, the state evolves to the sort of state we have in the homodyne experiment: a coherent superposition over different relative photon numbers with a well-defined phase. ${ }^{3}$ As argued previously, such a state shows interference despite the fact that the reduced density operators have no coherence. Thus, one can explain the interference of independent lasers without invoking coherence.

Factist: Hmm. I'm sure there's some example that demonstrates the need for coherence. Otherwise, how could you explain the fact that the predictions that were made on the basis of assuming coherent states were never found to be in error? I just need to think some more about it... What about the following case? [...]

[A long series of examples and fictionist explanations of these examples follows. ${ }^{4}$ Finally, the fictionist sees a pattern.]

\footnotetext{
3 The surprising result that two Fock states yield an interference pattern in the joint distribution of a multi-particle detection was first discovered in the context of Bose-Einstein condensates by Javanainen and Yoo 5|. The issue was investigated in the optical context by Mølmer [13], and a simple analytical investigation can be found in 35.

${ }^{4}$ See [21] for the fictionist response to many other standard interference experiments.
}

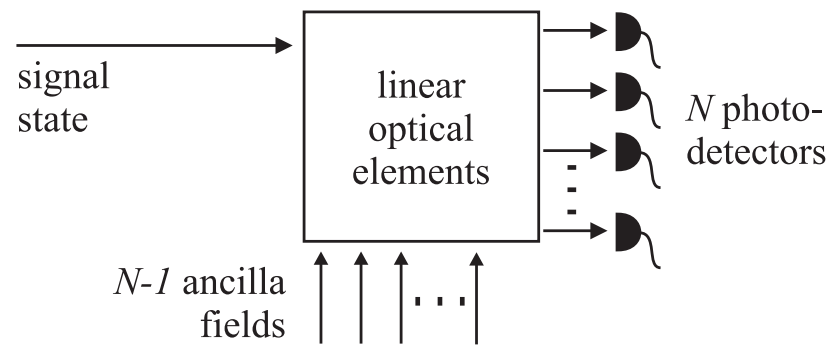

FIG. 3: A schematic for a general linear optical interferometer. The signal mode and $N-1$ probe fields are injected into a general linear optical interferometer, followed by photodetection on all $N$ output modes.

Fictionist: You can stop looking for more examples, because I have a general theorem that will deal with all linear optical experiments. The most general such experiment is a $2 N$-port interferometer, wherein one of the input ports corresponds to the signal field (the one which we are trying to identify as coherent or not), while the other $N-1$ correspond to independent probe fields, and at each of the $N$ output ports is a photodetector. The body of the interferometer may involve any combination of linear optical elements [see Fig. 3].

Now consider the following. If the photodetectors were ideal, so that together they constituted a measurement of the total number of photons in all modes, and if the optical elements were lossless, so that they conserved the total number of photons, and if the probe fields were described by number states, so that the total number of photons in the probe fields was known, then one could immediately infer the number of photons in the signal mode by taking the difference of the total number detected and the total number in the probe fields. Such an idealized interferometer would therefore constitute nothing more than a fancy measurement of the number basis on the signal field.

In practice, photodetectors constitute an error-prone measurement of the number basis [36], photons may be lost to absorption somewhere in the interferometer (which has the same effect as coupling into an output mode upon which a measurement of the number basis is performed but the outcome is unregistered), and each of the probe fields is, by Mølmer's argument, an incoherent mixture of number states. However, this simply means that there is uncertainty in the number of photons in the probe and uncertainty in the total number measured, and consequently that such an interferometer is simply an error-prone measurement of the number in the signal. But the statistics of such a measurement are still completely insensitive to any off-diagonal elements of the density operator in the number basis.

This result, by the way, explains why calculations wherein sources are represented by coherent states have agreed so well with experiments. The most general linear detection scheme (as described above) is completely in- 
sensitive to the values of the off-diagonal elements of the density matrix! One could assume any values whatsoever for these elements without affecting the result of such calculations. The use of coherent states in the place of Poissonian mixtures of number states will yield the correct predictions, and may even simplify the calculation, but they are simply a convenient fiction.

Factist: Fine. I grant that these sorts of experiments don't settle the issue in my favor. But they don't decide it in your favor either. All you've shown is that if you can't generate coherence, then you can't detect it either. But if you could generate it, then you could also detect it. I can prove this to you using the simple homodyne example from before where I imagine that the local oscillator comes from this hypothetical coherent source.

Let the state of the local oscillator be a coherent state $|\beta\rangle$. A simple calculation shows that the difference in number at the two output ports is

$$
\begin{aligned}
\operatorname{Tr}_{a b}\left[e^{i \phi N} \rho e^{-i \phi N} \otimes|\beta\rangle\langle\beta|\left(a^{\dagger} b+b^{\dagger} a\right)\right] & \\
& =\operatorname{Tr}_{a}\left[\rho\left(\beta^{*} e^{-i \phi} a+\beta e^{i \phi} a^{\dagger}\right)\right]
\end{aligned}
$$

Again, the only way one can obtain interference in this case is if $\rho$ has coherence between different number eigenstates. Therefore such a measurement is a test for the presence of coherence in the signal. So this shows that if you could generate coherence, somehow, then you could use it to detect coherence. So it ultimately just comes back to the issue of whether you can generate coherence, ...

Fictionist: But Mølmer's argument...

Factist: ... and I've now got a new idea for how to do it. Basically, we just downconvert from an EM field that is of sufficiently long wavelength. Take a radio wave as an extreme example. Surely radio waves are in coherent states because the way I generate them is by an oscillating current in an antenna rather than by stimulated emission in atoms. This current can be treated classically. It's just a charge moving up and down. [Emphatically waves a fist up and down to illustrate.] If you look in Jackson [37], you'll find that a classical oscillating current interacting with a quantum EM field generates a coherent state. Even microwaves can be generated by oscillating currents, so all I need to do to get a coherent state at optical frequencies is to downconvert from the microwave regime.

Fictionist: Even if you knew the time of the first peak of a microwave to some error that is small relative to the period of a microwave, this would be converted into an optical field with the same peak position and the same absolute error on the peak position, which is large compared to the period of an optical wave. This means that you would have complete ignorance of the optical phase, and thus no coherence.

Factist: Well, someday it should be technologically feasible to build antennae that have currents oscillating at optical frequencies. You have to admit that from that day onwards we would have optical sources that were genuinely coherent.

Fictionist: Would I? Hmmm. [Considers the question for a while.] It seems to me that even in the case of an oscillating current, I can think back to how it was made and see that one only ever gets entanglement, never coherence.

Factist: But then you would have to admit that microwaves and radio waves are not in coherent states either!

Fictionist: Yes, now that I think about it, that's right. So let me refine my thesis: coherence between number eigenstates isn't just a fiction for fields at optical frequencies, it's a fiction for fields of any frequency.

Factist: Ok... [exasperated] So how are you going to obtain the classical limit? Everyone knows that classical EM fields correspond to large-amplitude coherent states, and you do need to recover the classical limit for optical waves, microwaves, radio waves, and electric currents under the right conditions.

Fictionist: I suppose that we will simply have to rethink the notion of quantum-classical correspondence. Maybe there is some alternative way to obtain classical EM fields. I don't quite see it yet, I admit, but I'm confident it will work out.

Factist: This makes no sense to me. Why not assume coherence? Then you have no problems with the classical limit.

Fictionist: Look, even if you do assume that the electrons in an antenna are in a coherent superposition of energy eigenstates and therefore have a well defined phase, you don't know the phase. When you wave your fist up and down [repeats the motion], it suggests that you could directly know the phase of the electron motion, but even for radio waves it's too high in frequency for you to ever know it.

Factist: Let's not go back to that! You already conceded that there's nothing wrong with an ignorance interpretation of a proper mixture. I don't claim that anyone necessarily knows the phase, but simply that there is a well-defined phase.

Fictionist: Look, this whole conversation is starting to make my head hurt. The point is that all of my calculations come out right without the need to introduce coherences, so there's no reason to assume they exist. They are just metaphysical baggage.

Factist: I've also got a head-ache, all of my calculations also come out right, and I still think that you're wrong.

\section{A RESOLUTION}

The debate we presented was ultimately about whether the intrinsic properties of a system are best described by a coherent or an incoherent quantum state. But the whole debate presumes that quantum states only contain information about the intrinsic properties of a system. 
We submit that this presumption is mistaken; quantum states also contain information about the extrinsic properties of a system, that is, the relation of the system to other systems external to it, and whether or not coherences are applicable depends on the external system to which one is comparing.

The philosophical distinction between intrinsic and extrinsic properties can be explained as follows [38]:

A sentence or statement or proposition that ascribes intrinsic properties to something is entirely about that thing; whereas an ascription of extrinsic properties to something is not entirely about that thing, though it may well be about some larger whole which includes that thing as part. A thing has its intrinsic properties in virtue of the way that thing itself, and nothing else, is. Not so for extrinsic properties, though a thing may well have these in virtue of the way some larger whole is. The intrinsic properties of something depend only on that thing; whereas the extrinsic properties of something may depend, wholly or partly, on something else.

In the context of the argument of the dialogue, our claim is that the phase of an optical mode is an extrinsic property of that mode, only defined in relation to an external phase reference, and the fact that there are many possible choices for this phase reference is the source of the debate.

An analogy is useful here. In special relativity, if one is not careful, an apparent contradiction arises. Suppose Alice and Bob are observers with some non-zero relative velocity, and suppose we denote the length of Alice's metre stick by $L_{1}$ and the length of Bob's metre stick by $L_{2}$. Now, a simple application of length contraction would seem to suggest that Bob ought to conclude that $L_{1}<L_{2}$, while Alice ought to conclude that $L_{1}>L_{2}$. But these statements are contradictory! Who is right? Is it Alice's or Bob's metre stick that is longer? Surely, one might argue, there must be some matter of fact about which is longer. But the idea that either Alice or Bob must be right and the other wrong is simply mistaken. The mistake was in assuming that there is a single intrinsic property - the length of Alice's metre stick - to which they are both referring. Really, there is only the world-sheet of Alice's metre stick, and the world-sheet of Bob's metre stick, and different time-slices of these sheets. When Alice compares lengths she is comparing a particular pair of time-slices of these sheets, while when Bob does so, he is comparing a different pair of timeslices. Thus, if $L_{1}^{A}$ and $L_{2}^{A}$ denote the lengths of the time-slices compared by Alice, and $L_{1}^{B}$ and $L_{2}^{B}$ denote the lengths of the time-slices compared by Bob, we have $L_{1}^{A}>L_{2}^{A}$ and $L_{1}^{B}<L_{2}^{B}$ and no contradiction. The appearance of a contradiction is dispelled when one realizes that Alice and Bob were making claims about different entities.
We propose the same sort of resolution to the dispute between the factist and the fictionist. The factist's use of the coherent state $|\alpha\rangle=\sum_{n=0}^{\infty} \frac{e^{-|\alpha|^{2} / 2} \alpha^{n}}{\sqrt{n !}}|n\rangle$ and the fictionist's use of the incoherent state $\rho=$ $\sum_{n=0}^{\infty} \frac{e^{-|\alpha|^{2}}|\alpha|^{2 n}}{n !}|n\rangle\langle n|$ seem to be at odds with one another because there is a presumption that the two are describing the same degree of freedom, namely, the intrinsic properties of a single optical mode.

However, the dispute can be resolved if one grants that their quantum states describe the relation between this optical mode and an external phase reference, and if one recognizes that the factist and the fictionist are implicitly making use of different external phase references.

Denoting the factist and fictionist's external phase references by $R$ and $R^{\prime}$ respectively, we can make the point as follows: whereas the factist's $|\alpha\rangle$ concerns the relation between $S$ and $R$, the fictionist's $\rho$ concerns the relation between $S$ and $R^{\prime}$ ! They are describing different entities and so it is not a contradiction if their descriptions differ. In the following, we shall attempt to defend this point of view in more detail.

\section{PRELIMINARIES ABOUT REFERENCE FRAMES}

We begin with a few comments about reference frames (RFs). Because the example of a Cartesian RF is arguably more intuitive than that of a phase reference, we shall illustrate the central concepts with this example.

When a quantum state of a spin- $1 / 2$ system is assumed to be spin-up along the $\hat{z}$ direction, we are assuming the existence of a Cartesian reference frame, with respect to which the $\hat{z}$ direction is defined. We need not assume that this Cartesian frame is defined by Newton's absolute space, because we only ever compare the orientations of physical objects to other physical objects and never to any purported absolute space. Similarly, we only ever compare the phases of optical modes to other oscillating systems, and never to any purported absolute time standard, so we have no need in practice of an absolute time standard.

Consequently, a reference frame can in practice always be taken to be defined by some physical object. It follows that one ought to be able to apply quantum theory to the reference frame itself if one wishes. So in describing any given experimental situation, one is forced to make a choice about whether the RF is treated as external or as internal. To be precise, to treat an RF externally is to treat it as a background resource to which one's description of the system is referred. On the other hand, to treat it internally is to incorporate it into the formalism and to assign it degrees of freedom like any other physical system.

In quantum theory, treating an RF internally requires introducing a Hilbert space for it. Treating it externally usually implies that it is being treated classically. How- 
ever, for any theory, not just quantum theory, one can introduce a distinction between treating a reference system as part of the system under investigation and treating it as part of the background. For instance, in Newtonian mechanics, if we consider a ball bouncing off a wall, we may treat the wall either as an external potential or as a dynamical system that also obeys Newton's laws. We shall be arguing that neither method of representation is preferred; it is not as if one of these ways of treating a reference frame is correct and the other incorrect. It is simply a conventional choice of the physicist.

Another consequence of reference frames being defined by physical objects is that there can be many distinct physical systems that define reference frames for the same symmetry group. Thus, our spin- $1 / 2$ system may be known to have been generated by post-selecting the up outcome in a Stern-Gerlach experiment, so that it may be said to be in the quantum state $|+z\rangle$ relative to a $\hat{z}$-axis defined by the Stern-Gerlach magnet. However, any other Stern-Gerlach magnet also defines a $\hat{z}$-axis and if the two magnets are not aligned, the spin- $1 / 2$ system will not be described by the state $|+z\rangle$ relative to the second magnet. If the second magnet is related to the first by a rotation $\Omega \in S O(3)$, then the quantum state of the spin- $1 / 2$ system relative to the second magnet will be $R(\Omega)|+z\rangle$, where $R(\Omega)$ is a unitary representation of $\Omega$. If we consider a third magnet, for which the orientation to the first magnet is completely unknown, then we must average over $\mathrm{SO}(3)$ rotations with the uniform measure $d \Omega$ over $\mathrm{SO}(3)$, and the quantum state of the spin- $1 / 2$ system relative to the third magnet will be $\int d \Omega R(\Omega)|+z\rangle\langle+z| R(\Omega)^{\dagger}[39]$.

It is worthwhile to introduce a distinction between an $\mathrm{RF}$ that has some correlation with the system of interest, which we shall call an implicated RF, and one that is completely uncorrelated with the system, which we shall call a nonimplicated RF. In our example of the spin- $1 / 2$ system, the first and second magnets were implicated RFs while the third was a nonimplicated RF.

We now repeat the main point of our resolution to the controversy for this case. The factist's description of a system $S$ is of its relation with an external $\operatorname{Rf} R$ that is implicated, whereas the fictionist, who insists on internalizing $R$, describes $S$ in terms of its relation with an external RF $R^{\prime}$ that is nonimplicated. Because they are describing different entities, there is no contradiction if the quantum states they use differ. Fig. 目illustrates the two paradigms of descriptions.

\section{RECONSIDERING THE DIALOGUE ACCORDING TO OUR RESOLUTION}

In the dialogue, the factist and fictionist both made an assumption which we believe to be mistaken, namely, that their quantum states describe only intrinsic properties of a system. If this assumption is relaxed, then the differences in their convictions can be accounted for

\section{$\underline{\text { Factist }}$}

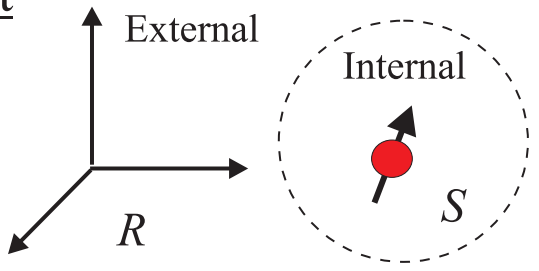

$\underline{\text { Fictionist }}$

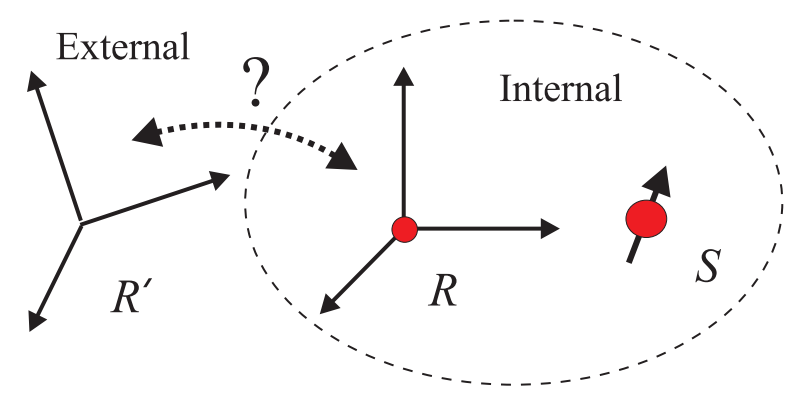

FIG. 4: Diagrammatic representation of the description of a system and reference frame. The factist treats the reference frame $R$ as external, whereas the fictionist treats the reference frame $R$ as internal and uncorrelated to any external reference frame $R^{\prime}$.

reasonably well by supposing that they are simply disagreeing about the $\mathrm{RF}$ with respect to which the extrinsic properties of the system are being defined. Essentially, the factist is inclined towards treating the RF to which the system is correlated as an external $\mathrm{RF}$, while the fictionist is inclined towards treating this RF internally, and leaving only a nonimplicated $\mathrm{RF}$ as external.

\section{A. Making sense of the states in the dialogue}

We shall consider the homodyne experiment described in the dialogue (but we start by focusing on the states rather than the measurements). Here, the system $S$ is the signal mode, and the reference frame $R$ is the local oscillator. When this is first discussed in the dialogue, the factist describes $S$ by the coherent state $|\alpha\rangle$, and treats $R$ as a classical field. Because $R$ has no representation in the quantum formalism, the factist is treating his phase reference externally. The fictionist, on the other hand, insists on treating $R$ within the quantum formalism. Consequently, $R$ is an internal phase reference for her. But the fictionist still implicitly makes use of an external phase reference, which we shall denote by $R^{\prime}$; it is just that the relation between $R^{\prime}$ and $R$ is assumed to be completely unknown.

(One might argue that the fictionist need not have an external phase reference at all. However, our fictionist agrees with the factist on the mathematical structure of the Hilbert space, including the formal possibility of co- 
herence, i.e., she agrees that phase is "in principle" an observable on this system. This suggests that she does possess an external phase reference but it is simply uncorrelated with $S$ or $R$.)

We now demonstrate that the incoherent state that the fictionist assigned to $S$, namely, the Poissonian mixture of number states of Eq. (5) is precisely how one would describe the relation between $S$ and external $R^{\prime}$ given that one describes the relation between $S$ and external $R$ by the coherent state $|\alpha\rangle$ of Eq. (11) and given that the relation between $R$ and $R^{\prime}$ is completely unknown. If $R^{\prime}$ was related to $R$ by the phase $\phi \in[0,2 \pi)$, and if the relation between $S$ and $R$ was described by the quantum state $\sigma$, the relation between $S$ and $R^{\prime}$ would be described by $U(\theta) \sigma U(\theta)^{\dagger}$, where $U(\theta)=e^{i \theta N}$ acts unitarily on the Fock space $\mathcal{H}_{S}$ of a single mode, and where $N$ is the number operator on $\mathcal{H}_{S}$ [40]. However, if one has no knowledge of the phase $\theta$ then one must average over all $\theta \in[0,2 \pi)$ [39], implying that the relation between $S$ and $R^{\prime}$ is described by

$$
\int_{0}^{2 \pi} \frac{d \theta}{2 \pi} U(\theta) \sigma U(\theta)^{\dagger}
$$

Thus, if we assume that the relation between $S$ and external $R$ is described by the factist's quantum state, $|\alpha\rangle\langle\alpha|$, the relation between $S$ and external $R^{\prime}$ ought to be described by the quantum state

$$
\begin{aligned}
\int_{0}^{2 \pi} \frac{d \theta}{2 \pi} U(\theta)|\alpha\rangle\langle\alpha| U(\theta)^{\dagger} & =\int_{0}^{2 \pi} \frac{d \theta}{2 \pi}\left|\alpha e^{i \theta}\right\rangle\left\langle\alpha e^{-i \theta}\right| \\
& =\sum_{n=0}^{\infty} \frac{e^{-|\alpha|^{2}}|\alpha|^{2 n}}{n !}|n\rangle\langle n|
\end{aligned}
$$

where the final equality is the familiar ambiguity of mixtures discussed in the dialogue. But this is precisely the quantum state assigned by the fictionist.

At one point in the dialogue, the factist argues that the reduced density operator on $S$ of the fictionist's quantum state for $R+S$ has a preferred convex decomposition into coherent states, and in this sense is consistent with the factist's quantum state for $S$. The fictionist rightly points out that the factist cannot simultaneously agree that $R$ and $S$ are entangled while maintaining that $S$ is in a pure state. By the lights of our account, the factist description is consistent with the fictionist's, but for a very different reason. What the factist describes as "the state of $S$ " is really the quantum state describing the relation between $S$ and $R$, and what the fictionist describes as "the state of $S$ " is really the quantum state describing the relation between $S$ and $R^{\prime}$. Therefore, if we want the fictionist's description of the relation between $S$ and $R$, we don't want what she calls "the reduced density operator on $S$ " nor any element of a convex decomposition thereof. What we really want is the quantum state on a Hilbert space that somehow encodes the relation between the Hilbert space she associates with $S$ and the Hilbert space she associates with $R$. As it turns out, it is the noiseless subsystems [41] with respect to phase rotations that serve this purpose. The states within these noiseless subsystems are coherent states in the limit of a large phase reference. See Ref. [42] for more details.

In the dialogue, the argument over coherence was often repeated "one level up", for instance, at the level of the gain medium of the laser rather than the field. Arguments at these higher levels can be understood in the same way as the arguments at the lower level. To see this, it is useful to redescribe what occurred in the dialogue in the light of our resolution.

After the initial argument over coherence of the system $S$ (the field), the factist mistakenly buys into the fictionist's argument and agrees to internalize $R$ (the gain medium), but he still insists on describing the pair relative to some external $R^{*}$ that is correlated to $R$ (for instance, the electrons of the pumping mechanism, considered as an external RF). The fictionist does not agree to allow $R^{*}$ as an external $\mathrm{RF}$ and so promptly internalizes it, and continues to describe everything relative to an uncorrelated RF $R^{\prime}$, which is left implicit in the discussion. Again, both descriptions are equally valid and differ only insofar as they relate the system under investigation to different external RFs.

As a specific example, we consider the disagreement that arises in the case where the RF $R$ is the local oscillator in the homodyne experiment. When the factist internalizes the local oscillator (which occurs when he makes the case that one can detect coherence given a coherent source), he describes it relative to an implicated external RF $R^{*}$. In the dialogue, the factist assumes an $R^{*}$ which is aligned perfectly with $R$. Thus, the factist describes the relation between $S$ and $R^{*}$ with precisely the same quantum state as he used to describe the relation between $S$ and $R$, namely, $|\alpha\rangle$. The factist describes the relation between $R$ and $R^{*}$ by a coherent state $|\beta\rangle$.

Now consider how to redescribe $R+S$ relative to a nonimplicated reference frame $R^{\prime}$. Let the system be realized by mode $a$ and the reference $R$ by mode $b$. Given that the relation between $R^{\prime}$ and $R^{*}$ is completely unknown, we must average the factist's quantum state over all phase rotations on $R+S$. Thus, the quantum state on modes $a$ and $b$ relative to $R^{\prime}$ is

$$
\rho_{a b}=\int_{0}^{2 \pi} \frac{d \theta}{2 \pi} V(\theta)|\alpha\rangle\langle\alpha|\otimes| \beta\rangle\langle\beta| V^{\dagger}(\theta),
$$

where we have defined the unitary operator $V(\phi)=$ $\exp \left(i \phi N_{b}\right) \exp \left(i \phi N_{a}\right)$, with $N_{a, b}$ the number operators for modes $a, b$. This state is equal to

$$
\rho_{a b}=\sum_{n} p_{n}\left|\psi_{n, \phi}\right\rangle\left\langle\psi_{n, \phi}\right|,
$$

where $p_{n}$ is a Poissonian distribution over $n$ and $\psi_{n, \phi}$ is defined in Eq. (13), where the parameter $T$ which appears therein is related to $\alpha$ and $\beta$ by $|\alpha|^{2} /|\beta|^{2}=$ $\left(1-T^{2}\right) / T^{2}$. This can be easily verified using the techniques of Ref. 21]. But this is precisely the state, 
Eq. (17), that is adopted by the fictionist to describe $R+S$.

Whenever a reference frame $R$ is internalized, one's description becomes relative to a new external reference frame. If, however, in the experiments of interest, the system $S$ is only ever compared to $R$, and no comparison of either is ever made to the new external reference frame, then for the predictions of the outcomes of such experiments, it makes no difference whether the new reference frame is implicated or not, and thus it makes no difference what the distribution over the global phase of $R+S$ is. For such experiments, the factist's and the fictionist's descriptions yield completely equivalent predictions.

Note, in addition, that whenever one internalizes a reference frame, one must choose a physical description (i.e., a quantum state) to represent what was previously described classically. Thus, one must deal with the myriad of issues associated with the quantization of a classical system. In particular, if it is demanded that the new description (with an internal RF) gives identical predictions as the previous description (with an external RF), the quantum state chosen must satisfy some conditions of a "classical limit". For example, when the factist is convinced by the fictionist that he should treat his local oscillator internally, he chooses to represent the local oscillator quantum mechanically as a coherent state $|\beta\rangle$ (relative to his new external phase reference $R^{*}$ ). It would seem necessary, then, that he takes the $|\beta| \rightarrow \infty$ limit in order to have complete agreement with his previous description. We will return to this issue in the next subsection, when we consider measurements.

\section{B. Making sense of the measurements in the dialogue}

To further our case, we show that the observable $b a^{\dagger}+b^{\dagger} a$ of Eq. (21) that the fictionist uses to describe the homodyne measurement is precisely the observable describing a measurement of the quadrature of the signal relative to the local oscillator when the latter is treated internally, all from the perspective of a nonimplicated external RF. We establish this equivalence in two steps.

First, we note that the observable $b a^{\dagger}+b^{\dagger} a$ on two modes $(a$ and $b)$ is the same with respect to any external phase reference, because it is invariant under "passive" phase shifts (that is, the unitary operator $V(\phi)=$ $\exp \left(i \phi N_{b}\right) \exp \left(i \phi N_{a}\right)$ as defined in the previous section). We say, then, that the observable $b a^{\dagger}+b^{\dagger} a$ only yields information about the relative degrees of freedom of $S+R$, because it is independent of any external RF. Moreover, any party with a nonimplicated RF claiming to measure only relative degrees of freedom we argue must use observables with this invariance property. ${ }^{5}$

\footnotetext{
5 The problem of determining the optimal measurements for
}

Second, we show that the fictionist reproduces the factist's predictions, which implies that the fictionist is also implementing a measurement of the quadrature of $S$ relative to $R$. Recalling that the factist describes the measurement by $\beta^{*} a+\beta a^{\dagger}$ and describes the relation between $S$ and the external $R$ (after the phase shifter) by the coherent state $\left|\alpha e^{-i \phi}\right\rangle$, it follows that he predicts a mean quadrature

$$
\left\langle\alpha e^{-i \phi}\left|\left(\beta^{*} a+\beta a^{\dagger}\right)\right| \alpha e^{-i \phi}\right\rangle=\beta^{*} e^{-i \phi} \alpha+\beta e^{i \phi} \alpha^{*} .
$$

The fictionist, on the other hand, describes $R+S$ relative to a nonimplicated external RF $R^{\prime}$ using $\rho_{a b}$ of Eq. (17), which can be written as a mixture of products of coherent states, as in Eq. (25). After the phase shifter, we have

$$
\rho_{a b}(\phi)=\int_{0}^{2 \pi} \frac{d \theta}{2 \pi} V(\theta)\left|\alpha e^{-i \phi}\right\rangle\left\langle\alpha e^{-i \phi}|\otimes| \beta\right\rangle\langle\beta| V^{\dagger}(\theta),
$$

Thus, the mean value of $a^{\dagger} b+a b^{\dagger}$ is

$$
\operatorname{Tr}\left[\rho_{a b}(\phi)\left(a^{\dagger} b+a b^{\dagger}\right)\right]=\beta^{*} e^{-i \phi} \alpha+\beta e^{i \phi} \alpha^{*} .
$$

This coincides with the factist value for the mean quadrature, so it is appropriate to say that the fictionist's measurement is indeed of the relative quadrature of $S$ to $R$.

Note that achieving this agreement did not require the amplitude of the coherent state $|\beta\rangle$ to be large. However, if all measurements (such as higher-order correlations of the photocurrents) are to be equivalent, one must take the limit $|\beta| \rightarrow \infty$. This would be required, for example, if one performed the above analysis using the formalism of generalized measurements (POVMs [46]) and demanded that the Born rule gave equivalent results in both descriptions. If $|\beta|$ was finite then there would be correction terms [4].

In the dialogue, the argument about whether it is possible to detect coherence ends with the factist concluding that "if one could generate coherence then one could detect it". What the factist really establishes with his argument is simply that if an internal RF $R$ (the local oscillator) is correlated with an external RF $R^{*}$ (so that the quantum state of the local oscillator has coherence), then by measuring the relation between $S$ and $R$ (through the homodyne detection), one obtains information about the relation between $S$ and $R^{*}$, in particular, whether they are correlated (and thus whether the quantum state of the signal was coherent or not).

Finally, it should be noted that if one is measuring only relations among the parts of the system, then the relation between the system and the external $\mathrm{RF}$ will be of no significance. Mathematically, such measurements are associated with incoherent POVMs, i.e., POVMs that

inferences about relative degrees of freedom is considered in Refs. 43, 44, 45] 
are invariant under collective phase rotations, so that the statistics are insensitive to the phase distribution of the state. This has been emphasized by Nemoto and Braunstein [20]. Notwithstanding this fact, it is always possible to make a measurement of the relation between the system and an external RF, and in this case the POVM is non-invariant under phase rotations and the phase distribution of the state has empirical significance. As an example, in the factist's original description of homodyne detection, where the quantum state describes the relation between the system and an external local oscillator, the use of a coherent state was necessary to obtain the correct predictions.

\section{Making sense of the transformations in the dialogue}

In the dialogue, the factist and the fictionist both described the phase shifter as a transformation of the intrinsic properties of the system. However, it is only the relation between the signal and local oscillator that is affected. This view is corroborated by the fact that the outcome of the measurement would be precisely the same if the phase shifter was instead placed in the path of the local oscillator mode. In other words, although active and passive transformations may be distinguished in the formalism, there is no physical distinction between them.

\section{Relative localization}

We can also understand the results on the interference of two lasers within our perspective. Consider one of the lasers to be the signal and the other to be the phase reference. To say that they are independent is to say that the phase reference is not correlated with the signal at the outset, i.e. nonimplicated in our terminology. The fact that interference can be achieved after sufficiently many photodetections demonstrates that the two become correlated over time. But this is precisely what one expects given that the homodyne detection implements a measurement of the relative phase of the two; the quantum state is updated to one that reflects the particular relative phase measured in the experiment. Note that the same conclusion could be reached if one of the lasers was treated externally (i.e. classically). The relation between the two modes would be described by a mixture of coherent states, and the phase distribution would evolve, as one accumulated data, from uniform to highly peaked about some random value. In other words, the external phase reference would evolve from nonimplicated to implicated. See [35] for further details.

\section{E. Microwaves, radio waves, and humanly-perceptible oscillations}

At the end of the dialogue, the fictionist argues against the factist's suggestion that upconversion from coherent sources at low frequencies could yield coherence at optical frequencies; this argument is originally due to Wiseman 23]. Thereafter, the factist appeals to a hypothetical optical frequency antenna, which leads the fictionist to deny that even radio waves or microwaves could be coherent. Essentially, the factist was appealing to the notion that a reference frame consisting of oscillating electrons is somehow more worthy to be left as an external RF than is a RF consisting of a high intensity EM field. The fictionist eventually decides to stick to his program of constant internalization of any RF and denies that even this sort of RF can be left external. ${ }^{6}$ There is only one holdout by the end of the dialogue, which is an observer's own sense of time. The fictionist essentially argues that the only thing that she is willing to take as an external RF would be a human's own internal clock. ${ }^{7}$ However, the choice of whether to treat any clock internally or externally is a conventional one; the clock provided by an observer's sense of time has no greater claim to being an external RF than any other.

\section{F. Applying the arguments in the dialogue to the whole universe}

The debate in the dialogue could have been continued to the point where the factist and fictionist were arguing about the existence of coherence in the initial state of the universe. Similarly, for our proposed resolution, one could ask what occurs when the entire universe is the quantum system of interest, such that there is no physical system left over to act as an external RF. To ask either of these questions is to presume that it makes sense to apply quantum theory to the universe as a whole. It appears to us highly likely, however, that quantum theory, as it is currently formulated, applies only to subsystems of the universe. This is not to say that we reject the idea of theories that apply to the universe as a whole; we only reject the idea that quantum theory as it stands is such a theory.

\footnotetext{
6 In some of the literature on this debate [16, 17, 25] there was an impression that an optical frequency antenna was somehow a more legitimate clock than a laser. The notion that the fictionist arguments apply equally well to electronic clocks as to optical clocks was first made by Wiseman [23].

7 The idea that this might be the only clock that a die-hard fictionist would allow to be external (and that the reason she would allow it is the persuasiveness of waving one's hand up and down!) is also due to Wiseman [23].
} 


\section{THE CONTROVERSY IN OTHER CONTEXTS}

We now consider how our arguments generalize to other contexts in which the quantum coherence controversy arises. We begin by noting the connection to superselection rules.

To assert that there is a selection rule for some quantity is to assert that this quantity is conserved. On the other hand, to assert that there is a superselection rule for a quantity is to assert the impossibility of preparing coherent superpositions of nondegenerate eigenstates of the associated Hermitian operator (more generally, the impossibility of preparing density operators with elements connecting nondegenerate eigenstates) [4].

The position of the fictionist can be restated as a belief in the existence of a superselection rule for total photon number. We have argued that whether to use a factist or fictionist description is a conventional choice that depends on one's choice of RF. This implies that whether to adopt a superselection rule for photon number is also a conventional choice that depends on one's choice of RF. More specifically, our claim is that a superselection rule for photon number is applicable if and only if one's external phase reference is nonimplicated.

It is tempting to think that this idea - that it is a conventional choice of the theorist that determines whether a superselection rule holds or not - can only be maintained in some contexts but not others. For instance, one might think that coherence between charge eigenstates is different in kind from coherence between photon number eigenstates.

However, we do not see any significant difference between photon number and other conserved quantities. Admittedly, it may be more difficult to construct good reference frames for some degrees of freedom, but there is nothing in principle preventing their construction. For instance, to lift the superselection rule associated with charge, one must simply have a large reference system with respect to which one can coherently exchange charge, as argued by Aharonov and Susskind 1]. As another example, the experimental realization of BoseEinstein condensation in alkali atoms provided a reference frame for the phase that is conjugate to atom number. When treating this RF externally, interference experiments with condensates must be interpreted in terms of states that are coherent superpositions of eigenstates of atom-number. We see no obstacle in principle to lifting more general sorts of superselection rules as well.

Similarly, one might think that there are conserved quantities for which a superselection rule is never applicable. For instance, the case of linear momentum may appear to be different in kind from that of photon number, because a superselection rule for linear momentum would seem to imply that objects could not be localized in space, and this, one might think, would be contrary to what is observed. However, all that is ever observed is the localization of systems relative to other systems. If we treat these reference systems internally, and refer our systems to a nonimplicated external RF, then we have a paradigm of description wherein there is no coherence between eigenstates of total linear momentum, and thus a superselection rule for the latter, while relative localization is still achieved. Such cases are not different in principle from any other conserved quantity. What sets them apart in practice is the ubiquitous nature of the associated reference systems, such as those for spatial location. The more ubiquitous a RF, the more theorists seem inclined to treat it externally, but an internalized treatment is just as valid.

Several recent papers have considered the problem of internalizing reference frames. Specifically, it has been shown how to internalize a Cartesian reference frame for spin systems [49], a clock for spin systems [49, 51], and a clock for oscillators [50]. A particular strategy for internalization of general reference frames can be found in Ref. [52].

\section{NEITHER PARTY WINS THE DEBATE}

In the end, which of our two protagonists can claim to have won the debate? Ultimately, treating one view as superior is only justified if the alternative can be shown to lead to factual errors, or to descriptions of such complexity that - as for Simplicio and Segredo in Galileo's Dialogue - Occam's razor can be brought to bear.

We have argued that if reference frames treated internally are given "classical limit" descriptions compatible with an externalized treatment, then the factist and the fictionist give identical predictions for all experiments. Thus, neither party's state assignments can be held up as more empirically accurate than the other's. (In as much as this debate captures many aspects of the controversy about continuous variable quantum teleportation $16,17,18,19,20,22,23,26]$, we believe that neither position in this controversy, factist or fictionist, is more correct than the other. See also [53] for further insight into the issue of the entanglement resource in continuous variable quantum teleportation.)

Can we use Occam's razor to favor one description over the other? The factist's description makes use of a smaller Hilbert space, and thus in many situations may be more efficient, especially when numerical calculations are required.

However, as mentioned, a limitation of our analysis is that we can assert the empirical equivalence of the two viewpoints only in the case where the physical systems comprising the reference frame have consistent physical descriptions from both the factist and fictionist points of view. In particular, this has necessitated the use of large amplitude coherent states in describing the local oscillators of the homodyne detection; these states are, in the large amplitude limit, "perfect" reference frames.

However, physical reference frames are never perfect they are finite, suffer back action and drift over time. It 
may therefore appear "more correct" to treat such RFs quantum mechanically, and thus view the fictionist's description as superior to that of the factist.

Nonetheless, it seems to us quite plausible that the imperfection of physical reference frames may be taken into account within the externalized mode of description of the factist by making use of mixed rather than pure states, generalized measurements rather than projective ones, and quantum operations rather than unitaries. ${ }^{8}$ For instance, Tyc and Sanders [47] have determined the generalized measurement for homodyne detection using a finite local oscillator. Note, however, that such descriptions, if they exist, are likely to be justified theoretically by first considering a fully quantum-mechanical model of the reference frame.

Thus, one of the positive outcomes of this debate and the resolution presented herein is that we have identified a set of interesting problems for future research: first, to determine the effects of various imperfections in one's $\mathrm{RF}$ within the fully quantum-mechanical treatment of the fictionist paradigm, and second, to determine how these effects can be modelled within the more economical paradigm of the factist by generalized quantum operations. These problems are significant insofar as imperfect reference frames may pose a challenge to achieving the fine control that is required for the successful implementation of quantum information processing protocols.

\section{Acknowledgments}

The authors gratefully acknowledge Barry Sanders, John Sipe, and Howard Wiseman for many long discussions on these matters, and Sam Braunstein, Florian Girelli, Netanel Lindner, David Poulin, Lana Sheridan, Danny Terno and Howard Wiseman for their thoughtful comments on a draft of this paper. S.D.B. is supported by the Australian Research Council, and T.R. is supported by the UK Engineering and Physical Sciences Research Council.

\section{APPENDIX A: A COMPARISON WITH ALTERNATIVE RESPONSES}

Wiseman 23] was the first to have the key insight that electronic and optical clocks are equally valid as phase references. Nonetheless, he still insisted on internalizing his clocks, and, unwilling to acknowledge any further external phase reference, he was pushed to justify the use of a coherent state for his clock by claiming that "...if the laser itself is the clock, then by definition it is coherent with respect to itself, so that it should be described by

\footnotetext{
${ }^{8}$ It may also be necessary to use a Hilbert space with superselection sectors.
}

a pure [coherent state] of zero phase". By the light of our analysis, this is a mistake. No quantity can be defined relative to itself. In particular, coherences describe relations - and as such are only defined between distinct systems.

The following are valid treatments of the clock: (1) the clock is treated as an external RF, in which case it is not described by a quantum state and therefore the issue of whether this state is coherent or not does not arise; (2) the clock is treated as an internal RF and the external $\mathrm{RF}$ with respect to which it is described is implicated, in which case one can justify the use of a coherent state for the internalized clock; (3) the clock is treated as an internal RF but the external RF with respect to which it is described is nonimplicated, in which case one must use an incoherent state to describe the internalized clock. When coherence appears in the state of an internal RF it is with respect to an external implicated $\mathrm{RF}$, not with respect to itself.

Another response to the controversy, advocated by van Enk and Fuchs [54], is that coherent states play a privileged role in the description of the propagating laser output state by making use of the quantum di Finetti theorem. Their arguments are compatible with the fictionist's description, as the state they ascribe the laser has no global coherence (when all modes are included). They argue that complete measurements on some of the propagating modes relative to some external classical RF (the example they use is a microwave field) will result in the remaining modes of the system becoming correlated in phase to this external RF. In our language, their argument is that the fictionist can perform complete measurements on part of the system relative to her nonimplicated $\mathrm{RF}$, correlating the systems and thus implicating the external RF. Thus, their argument is essentially that the fictionist could, in principle, perform measurements to implicate her external RF and, in so doing, move to a factist description.

\section{APPENDIX B: THE EPISTEMIC VIEW OF QUANTUM STATES}

A final caveat is in order. When we say "coherent quantum states concern extrinsic properties", we do not mean to suggest that the quantum states of some relational degree of freedom are in one-to-one correspondence with the different possible values that this degree of freedom may take, that is, the different possible physical states for that relational degree of freedom. We oppose this view, and believe that it is far more likely that the quantum states of a degree of freedom are in oneto-one correspondence with the different possible states of knowledge that one can have about the value of that degree of freedom, even though a satisfactory interpretation of the quantum formalism along these lines has yet to be provided. (See Refs. 54, 55] for discussions of this research program.) However, the argument we presented 
here does not really depend on which of these two views - the ontic or the epistemic view - one takes towards quantum states. Whether quantum states describe states of reality or states of knowledge about reality, we argue that they do not simply concern intrinsic properties but extrinsic properties as well.
[1] Y. Aharonov and L. Susskind, Phys. Rev. 155, 1428 (1967).

[2] P. W. Anderson, in The Lesson of Quantum Theory, J. D. Boer, E. Dal, O. Ulfbeck, Eds. (Elsevier, Amsterdam, 1986), pp. 2333.

[3] R. Haag, Il Nuovo Cimento, XXV, 2695 (1962).

[4] D. Kershaw and C. H. Woo, Phys. Rev. Lett. 33, 918 (1974).

[5] J. Javanainen and S. M. Yoo, Phys. Rev. Lett. 76, 161 (1996).

[6] W. Hoston and L. You, Phys. Rev. A 53, 4254 (1996).

[7] S. M. Yoo, J. Ruostekoski and J. Javanainen, J. Mod. Opt., 44, 1763 (1997).

[8] Y. Castin and J. Dalibard, Phys. Rev. A 55, 4330 (1997).

[9] M. R. Andrews, C. G. Townsend, H. -J. Miesner, D. S. Durfee, D. M. Kurn, W. Ketterle, Science 275, 637 (1997).

[10] L. Hardy, Phys. Rev. Lett. 73, 2279 (1994).

[11] D. M. Greenberger, M. A. Horne, and A. Zeilinger, Phys. Rev. Lett. 75, 2064 (1995).

[12] L. Hardy, Phys. Rev. Lett. 75, 2065 (1995).

[13] K. Mølmer, Phys. Rev. A 55, 3195 (1997); J. Mod. Opt. 44, 1937 (1997).

[14] J. Gea-Banacloche, Phys. Rev. A 58, 4244 (1998).

[15] K. Mølmer, Phys. Rev. A 58, 4247 (1998).

[16] T. Rudolph and B. C. Sanders, Phys. Rev. Lett. 87, 077903 (2001).

[17] S. J. van Enk and C. A. Fuchs, Phys. Rev. Lett. 88, $027902(2002)$

[18] T. Rudolph and B. C. Sanders, arXiv:quant-ph/0112020 (2001).

[19] S. J. van Enk and C. A. Fuchs, Quantum Inform. Compu. 2, 151 (2002).

[20] K. Nemoto and S. L. Braunstein, arXiv:quant-ph/0207135 (2002); Phys. Rev. A 68, 042326 (2003); Phys. Lett. A, 333, 378 (2004).

[21] B. C. Sanders, S. D. Bartlett, T. Rudolph, P. L. Knight, Phys. Rev. A 68, 042329 (2003)

[22] H. M. Wiseman, J. Mod. Opt. 50, 1797 (2003); arXiv:quant-ph/0104004 (2001).

[23] H. M. Wiseman, J. Opt. B 6, S849 (2004).

[24] M. Fujii, Phys. Rev. 68, 050302(R) (2003).

[25] R. W. Spekkens and J. E. Sipe, in Coherence and Quantum Optics VIII, eds. N. Bigelow et al. (Kluwer Academic, New York, 2003) p. 465.

[26] J. Smolin, arXiv:quant-ph/0407009 (2004).

[27] E. Schrödinger, Proc. Camb. Phil. Soc., 31, 555 (1935); Proc. Camb. Phil. Soc., 32, 446 (1936).

[28] L.P. Hughston, R. Jozsa, and W.K. Wootters, Phys. Lett. A 183, 14 (1993).
[29] B d'Espagnat, Conceptual Foundations of Quantum Mechanics, 2nd Ed. (Addison-Wesley, 1976).

[30] V. DeGiorgio and M. O. Scully, Phys. Rev. A 2, 1170 (1970).

[31] R. Graham, Progress in Optics XII, 233 (1974).

[32] U. Leonhardt, Measuring the Quantum State of Light, Cambridge Studies in Modern Optics, (Cambridge University Press, New York, 1997).

[33] D. F. Walls and G. J. Milburn, Quantum Optics (Springer-Verlag, Berlin, 1994).

[34] R. L. Pfleegor and L. Mandel, Phys. Lett. A 24, 766 (1967); Phys. Rev. 159, 1084 (1967); J. Opt. Soc. Am. 58, 946 (1968).

[35] H. Cable, P. L. Knight and T. Rudolph, Phys. Rev. A 71, 042107 (2005).

[36] W. Vogel and J. Grabow, Phys. Rev. A 47, 4227 (1993).

[37] J.D. Jackson, Classical Electrodynamics, 3rd Ed. (Wiley, 1998).

[38] D. Lewis, Philosophical Studies 44, 197 (1983).

[39] S. D. Bartlett, T. Rudolph and R. W. Spekkens, Phys. Rev. Lett. 91, 027901 (2003).

[40] S. van Enk, Phys. Rev. A 71, 032339 (2005).

[41] E. Knill, R. Laflamme and L. Viola, Phys. Rev. Lett. 84, 2525 (2000).

[42] S. D. Bartlett, T. Rudolph and R. W. Spekkens, Phys. Rev. A 70, 032307 (2004).

[43] S. D. Bartlett, T. Rudolph and R. W. Spekkens, Phys. Rev. A 70, 032321 (2004).

[44] N. Lindner, P. Scudo, and D. Bruss, arXiv:quant-ph/0506223 (2005).

[45] N. Gisin and S. Iblisdir, arXiv:quant-ph/0507118 (2005).

[46] M. A. Nielsen and I. L. Chuang, Quantum Computation and Quantum Information, (Cambridge University Press, Cambridge, 2000).

[47] T. Tyc and B. C. Sanders, J. Phys. A 37, 7341 (2004).

[48] G. C. Wick, A. S. Wightman and E. P. Wigner, Phys. Rev. 88, 101 (1952).

[49] D. Poulin, arXiv:quant-ph/0505081 (2005).

[50] G. J. Milburn and D. Poulin, arXiv:quant-ph/0505175 (2005).

[51] D. N. Page and W. K. Wootters, Phys. Rev. D 27, 2885 (1983).

[52] A. Kitaev, D. Mayers and J. Preskill, Phys. Rev. A 69, 052326 (2004).

[53] S. D. Bartlett, A. C. Doherty, R. W. Spekkens, and H. M. Wiseman, arXiv:quant-ph/0412158 (2004).

[54] C. H. Fuchs, arXiv:quant-ph/0205037 (2002); J. Mod. Opt. 50, 987 (2003).

[55] R. W. Spekkens, arXiv:quant-ph/0401052 (2004). 